\title{
The Interactive Antimicrobial Activity of Glycyrrhiza glabra L. Root Extracts and Conventional Antibiotics Against some Bacterial Triggers of Autoimmune Inflammatory Diseases
}

\author{
Haydon Maas ${ }^{1}$, Ian Edwin Cock ${ }^{1,2^{*}}$ \\ 'School of Natural Sciences, Nathan Campus, Griffith University, 170 Kessels Rd, Nathan, Queensland 4111, AUSTRALIA. \\ 2Environmental Futures Research Institute, Nathan Campus, Griffith University, 170 Kessels Rd, Nathan, Queensland 4111, AUSTRALIA.
}

\begin{abstract}
Background: Roots from Glycyrrhiza glabra L. are known for their antiinflammatory and antimicrobial properties. This study focuses on the growth inhibitory activity of $G$. glabra root extracts against some bacterial triggers of autoimmune inflammatory disease alone and in combination with conventional antibiotics. Methods: G. glabra root powder was extracted with solvents of varying polarity and screened for bacterial growth inhibition by disc diffusion assay. The minimum inhibitory concentration (MIC) was quantified by both liquid dilution and disc diffusion techniques. To screen for combinatorial effects, the G. glabra root extracts were combined with a range of conventional antibiotics and tested against each bacterium using liquid dilution assays. Where synergy was detected, the optimal ratios were determined using isobologram analysis. Toxicity was examined using an Artemia nauplii and HDF bioassays. Results: G. glabra root methanolic, aqueous and ethyl acetate extracts displayed antimicrobial activity against bacterial triggers of some autoimmune inflammatory diseases. The ethyl acetate extract was particularly potent, with MIC values $<500 \mu \mathrm{g} / \mathrm{mL}$ against $K$. pneumoniae and $A$. baylyi. The aqueous extract was also a moderate inhibitor of $A$. baylyi. The methanolic extract had moderate inhibitory activity against all bacteria except $P$. aeruginosa. Of further note, the aqueous extract interacted synergistically in combination with chloramphenicol against $K$. pneumoniae ( $\Sigma$ FIC 0.49). All extracts were nontoxic in the Artemia and HDF toxicity assays, further indicating their potential for
\end{abstract}

medicinal use. Conclusion: The G. glabra ethyl acetate root extract was a strong inhibitor of the growth of $K$. pneumoniae and $A$. baylyi and therefore has potential in the prevention and treatment of ankylosing spondylitis and multiple sclerosis. In addition, the aqueous root extract potentiated the inhibitory activity of chloramphenicol against a chloramphenicol resistant $K$. pneumoniae strain. Although the mechanisms of synergy are still unclear, compounds within the G. glabra root extracts may mimic the actions of resistance modifying agents. Isolation of these agents may be beneficial in antibiotic drug design against bacterial triggers of ankylosing spondylitis. Key words: Licorice, Synergy, Multi-drug resistant bacteria, Combinational therapies, Rheumatoid arthritis, Ankylosing spondylitis, Multiple sclerosis.

\section{Correspondence:}

Dr. I. E. Cock,

${ }^{1}$ Environmental Futures Research Institute, Nathan Campus, Griffith Univer-

sity, 170 Kessels Rd, Nathan, Queensland 4111, AUSTRALIA.

${ }^{2}$ School of Natural Sciences, Nathan Campus, Griffith University, 170 Kessels Rd, Nathan, Queensland 4111, AUSTRALIA.

Phone no: +61737357637

E-mail: I.Cock@griffith.edu.au

DOI: $10.5530 /$ pc.2018.2.14

\section{INTRODUCTION}

The discovery of penicillin by Alexander Fleming in 1929 was one of the greatest discoveries in the medical field and changed the way bacterial infections were treated. Since that discovery, medical science has focussed on microbially derived antibiotic agents to provide the majority of our first-generation drugs. However, despite many great advances in the treatment of pathogens, bacteria have developed resistance to all of the antibiotics commonly used clinically. ${ }^{1}$ In recent years, there has been an increase in the incidence of bacterial antibiotic resistance and several strains of medicinally important bacterial pathogens are now either extremely (XDR) or totally drug resistant (TDR). ${ }^{1}$ There are now limited therapeutic options for the diseases caused by these pathogens. This problem is expected to worsen in the future as bacteria exchange resistance genes and more strains become multi-drug resistant (MDR). The development of alternative antibacterial treatment modalities has become crucial and is considered by the World Health Organisation (WHO) to be one of the most serious challenges facing medical science. ${ }^{2}$ For several reasons reviewed elsewhere ${ }^{1}$ it is unlikely that the previous methods of antibiotic discovery/development will be as successful in the future and new treatment modalities are urgently required.

Traditional medicines and herbal remedies have great potential for antimicrobial drug development and there has recently been a substantial increase in interest in this field..$^{3-5}$ Glycyrrhiza glabra L. (licorice) root has been used for thousands of years in folk medicine to treat a wide variety of ailments. ${ }^{6,7}$ Whilst G. glabra root is best known as an effective treatment for peptic ulcers, it is also useful in treating hepatitis $\mathrm{C}$, as well as respiratory infections, gastric disease, peptic ulcers and inflammation. ${ }^{8}$ The phytochemistry of G. glabra has been relatively well reported. The root is rich in triterpenoid saponins, accounting for up to $20 \%$ of the dried mass. ${ }^{8}$ Of the saponins, glycyrrhizic and glycyrrhizinic acids are the most prevalent and are believed to contribute to many of the therapeutic properties associated with G. glabra root. The root is also rich in flavonoids, isoflavones, coumarins and stilbenoids. ${ }^{8}$

G. glabra extracts have reported to have been potent antibacterial activity against several human pathogenic bacteria. ${ }^{9}$ Further studies isolated several flavonoids from antibacterial extracts and demonstrated that they are effective against methicillin resistant Staphylococcus aureus (MRSA). ${ }^{10,11}$ Furthermore, those authors also reported that the G. glabra extracts restored the activity of oxacillin and several other $\beta$-lactam antibiotics against MRSA. Another study isolated glabridin, glabrene and licochalcone A from G. glabra and reported that they inhibited Helicobacter pylori growth in vitro. ${ }^{12,13}$ Further studies have reported that aqueous-ether G. glabra extracts inhibited the growth of a panel of bacteria including Eschericia coli, Bacillus subtilis and Staphylococcus aureus. ${ }^{14}$ Despite these previous reports, no studies have been yet screened G. glabra extracts for the ability to inhibit the growth of the bacterial triggers of autoimmune inflammatory diseases. Previous studies that identified the bacterial triggers of some autoimmune inflammatory diseases in genetically susceptible humans have allowed drug therapies targeting the initiating events of these diseases, thereby providing prophylactic chemotherapeutic options. Acinetobacter baylyi and Pseudomonas aeruginosa, were identified as bacterial triggers for multiple sclerosis, 
Klebsiella pneumoniae has been linked to ankylosing spondylitis and Proteus mirabilis was found to be a bacterial trigger of rheumatoid arthritis. ${ }^{15,16}$ This study aimed to investigate the growth inhibitory activity of G. glabra root extracts against P. mirabilis, K. pneumonia, A. baylyi and $P$. aeruginosa, alone and in conjunction with conventional antibiotics to evaluate their interactive effects.

\section{MATERIALS AND METHODS}

\section{Plant material and extraction}

The Glycyrrhiza glabra L. root used in this study was sourced from verified trees in Turkey by Noodles Emporium, Australia and supplied as a dried and ground powder. A voucher sample (GGR2016alic) has been stored at the School of Natural Sciences, Griffith University, Australia. Individual $1 \mathrm{~g}$ quantities of the material were weighed into separate tubes and $50 \mathrm{~mL}$ of methanol, deionised water, chloroform, hexane or ethyl acetate were added. All solvents were obtained from Ajax, Australia and were AR grade. The ground plant materials were individually extracted in each solvent for $24 \mathrm{~h}$ at $4^{\circ} \mathrm{C}$ with gentle shaking. The extracts were subsequently filtered through filter paper (Whatman No. 54) under vacuum, followed by drying by rotary evaporation in an Eppendorf concentrator 5301. The resultant extracts were weighed and redissolved in $10 \mathrm{~mL}$ deionised water (containing $1 \%$ DMSO).

\section{Qualitative phytochemical studies}

Phytochemical analysis of the G. glabra root extracts for the presence of saponins, phenolic compounds, flavonoids, phytosterols, triterpenoids, cardiac glycosides, anthraquinones, tannins and alkaloids were conducted by previously described assays. ${ }^{17-19}$

\section{Antibacterial screening Conventional Antibiotics}

Penicillin-G (potency of 1440-1680 $\mu \mathrm{g} / \mathrm{mg}$ ), chloramphenicol ( $\geq 98 \%$ purity by HPLC, erythromycin (potency $\geq 850 \mu \mathrm{g} / \mathrm{mg}$ ), gentamicin (potency of $600 \mu \mathrm{g} / \mathrm{mg}$ ), and tetracycline ( $\geq 95 \%$ purity by HPLC) were purchased from Sigma-Aldrich, Australia and were used as controls for the microplate liquid dilution assay. All antibiotics were prepared in sterile deionised water at stock concentrations of $0.01 \mathrm{mg} / \mathrm{mL}$ and stored at $4^{\circ} \mathrm{C}$ until use. For the disc diffusion studies, penicillin $(20 \mu \mathrm{g})$, nystatin (100 Units), ciprofloxacin $(2.5 \mu \mathrm{g})$ and chloramphenicol discs $(10 \mu \mathrm{g})$ standard discs were obtained from Oxoid Ltd., Australia and used as positive controls.

\section{Bacterial cultures}

All bacterial strains were selected based on their ability to trigger autoimmune inflammatory diseases in genetically susceptible individuals. ${ }^{15}$ Reference strains of Proteus mirabilis (ATCC21721), Klebsiella pneumoniae (ATCC31488), Acinetobacter baylyi (ATCC33304) and Pseudomonas aeruginosa (ATCC39324) were purchased from American Type Culture Collection, USA. All bacteria were cultured in nutrient broth (Oxoid Ltd., Australia). Streak nutrient agar (Oxoid Ltd., Australia) plates were tested in parallel to ensure the purity of all bacterial cultures and for sub-culturing. All bacterial cultures were incubated at $37^{\circ} \mathrm{C}$ for $24 \mathrm{~h}$ and were subcultured and maintained in nutrient broth at $4^{\circ} \mathrm{C}$ until use.

\section{Evaluation of antibacterial activity}

Antibacterial activity screening of the G. glabra root extracts was assessed using a modified disc diffusion assay. ${ }^{20,21}$ Penicillin $(20 \mu \mathrm{g})$, nystatin (100 Units), ciprofloxacin $(2.5 \mu \mathrm{g})$ and chloramphenicol discs $(10 \mu \mathrm{g})$ were obtained from Oxoid Ltd., Australia and used as positive controls to compare antibacterial activity. Filter discs infused with $10 \mu \mathrm{L}$ of distilled water were used as a negative control.

\section{Minimum inhibitory concentration (MIC) determination}

The minimum inhibitory concentration for each extract was determined using two methods. A liquid dilution MIC assay was employed as it is generally considered the most sensitive bacterial growth inhibitory assay. ${ }^{22}$ Furthermore, as microplate liquid dilution MIC assays are perhaps the most commonly used method of quantifying bacterial growth inhibition efficacy, use of this method allows for comparisons with other studies. A solid phase agar disc diffusion assay was also used in this study for comparison.

\section{Microplate liquid dilution MIC assay}

The MICs of the extracts were evaluated by standard methods. ${ }^{22,23}$ All plates were incubated at $37^{\circ} \mathrm{C}$ for $24 \mathrm{~h}$. P-Iodonitrotetrazolium violet (INT) was obtained from Sigma-Aldrich, Australia and dissolved in sterile deionised water to prepare a $0.2 \mathrm{mg} / \mathrm{mL}$ INT solution. A $40 \mu \mathrm{L}$ volume of this solution was added into all wells and the plates were incubated for a further $6 \mathrm{~h}$ at $30^{\circ} \mathrm{C}$. Following incubation, the MIC was visually determined as the lowest dose at which colour development was inhibited.

\section{Disc diffusion MIC assay}

The minimum inhibitory concentrations (MIC) of the extracts was also evaluated by disc diffusion assay as previously described. ${ }^{24}$ Graphs of the zone of inhibition versus $\ln$ concentration were plotted and MIC values were determined using ln linear regression.

\section{G. glabra fruit extract-conventional antibiotic synergy studies Fractional inhibitory concentration (FIC) assessment}

Interactions between the G. glabra root extracts and the conventional antibiotics were examined by determination of the sum of fractional inhibitory concentrations ( $\Sigma$ FIC) for each combination. ${ }^{4}$ The FIC values for each component ( $\mathrm{a}$ and $\mathrm{b}$ ) were calculated using the following equations where a represents the plant extract sample and $b$ represents the conventional antibiotic:

$$
\begin{aligned}
& \operatorname{FIC}(\mathrm{a})=\left(\frac{\text { MIC }[\mathrm{a} \text { in combination with } \mathrm{b}]}{\text { MIC }[\mathrm{a} \text { independently }]}\right) \\
& \operatorname{FIC}(\mathrm{b})=\left(\frac{\text { MIC }[\mathrm{b} \text { in combination with } \mathrm{a}]}{\text { MIC }[\mathrm{b} \text { independent }]}\right)
\end{aligned}
$$

The $\Sigma$ FIC was then calculated using the formula $\Sigma$ FIC = FIC (a) + FIC (b). The interactions were classified as synergistic $(\Sigma$ FIC $\leq 0.5)$, additive ( $\Sigma$ FIC $>0.5-1.0)$, indifferent $(\Sigma \mathrm{FIC}>1.0-4.0)$ or antagonistic $(\Sigma \mathrm{FIC}>4.0){ }^{23}$

\section{Varied ratio combination studies (isobolograms)}

For each combination producing synergistic interactions, nine different ratios spanning the range 10:90 (extract: antibiotic) to 90:10 (extract: antibiotic) were tested. All combinations were tested in duplicate in three independent experiments, providing six replicates for each combination ratio. The data is presented as the mean of six replicates. Data points for each ratio examined were plotted on a isobologram and this was used to determine optimal combination ratios to obtain synergy. Data points on or below the 0.5:0.5 line indicated synergy; those above the 0.5:0.5 line, up to and including the 1.0:1.0 line indicated an additive interaction; data points above the 1.0:1.0 line indicated indifferent interaction. 


\section{Toxicity screening}

Two assays were used to assess the toxicity of the individual samples. The Artemia nauplii lethality assay (ALA) was utilised for rapid preliminary toxicity screening, whereas the MTS cellular proliferation assay was used to determine a cellular evaluation of toxicity.

\section{Artemia franciscana Kellogg nauplii toxicity screening}

Potassium dichromate $\left(\mathrm{K}_{2} \mathrm{Cr}_{2} \mathrm{O}_{7}\right)$ (AR grade, Chem-Supply, Australia) was prepared in deionised water $(4 \mathrm{mg} / \mathrm{mL})$ and serially diluted in artificial seawater for use as a reference toxin. Toxicity of the G. glabra root extracts, the reference toxin and the conventional antibiotics was assessed using a modified Artemia franciscana nauplii lethality assay. ${ }^{25,26}$ The $\mathrm{LC}_{50}$ with 95\% confidence limits for each treatment was calculated using probit analysis.

\section{Cellular viability assay}

The G. glabra root extracts and conventional antibiotics were screened individually towards normal human primary dermal fibroblasts (HDF) as previously described. ${ }^{27} \mathrm{HDF}$ cells were obtained from American Type Culture Collection (ATCC PCS-201-012). The cells were cultured and maintained in Dulbecco's modified eagle medium (DMEM; Thermo Fisher Scientific, Australia), supplemented with $10 \%$ foetal calf serum (Life Technologies), $50 \mu \mathrm{g} / \mathrm{mL}$ streptomycin (Sigma-Aldrich, Australia) and $50 \mathrm{IU} / \mathrm{mL}$ penicillin (Sigm-Aldricha, Australia). Suspensions of cells which had obtained $80 \%$ confluency were resuspended in fresh media (lacking streptomycin and penicillin supplementation) and $70 \mu \mathrm{L}$ aliquots (containing approximately 5000 cells) were added to individual wells of a 96 well plate. A volume of $30 \mu \mathrm{L}$ of the test extracts or cell media (for the negative control) was subsequently added to individual wells and the plates were incubated at $37^{\circ} \mathrm{C}, 5 \% \mathrm{CO}_{2}$ for 24 hours in a humidified atmosphere. All extracts were screened at $200 \mu \mathrm{g} / \mathrm{mL}$. The cells were then washed in PBS ( $\mathrm{pH}$ 7.2) to remove interference due to sample colour. A volume of $20 \mu \mathrm{L}$ of Cell Titre 96 Aqueous One solution (Promega) was subsequently added to each well and the plates were incubated for a further $3 \mathrm{~h}$. Absorbances were recorded at a test wavelength of $540 \mathrm{~nm}$ and a blank wavelength of 690nm using a Molecular Devices, Spectra Max M3 plate reader. All tests were performed in at least triplicate and triplicate controls were included on each plate. The $\%$ cellular viability of each test was calculated using the following formula:

$\%$ cellular viability $=\frac{\text { Abs test sample }-(\text { mean Abs control }- \text { mean Abs blank })}{(\text { mean Abs control }- \text { mean Abs blank })}$

Cellular viability $\leq 50 \%$ of the untreated control indicated toxicity, whereas extracts or controls with $>50 \%$ untreated control viability were deemed to be nontoxic.

\section{Statistical analysis}

Data are expressed as the mean \pm SEM of at least three independent experiments. One-way ANOVA was used to calculate differences between the control and treated groups, with a $P$ value $<0.01$ considered to be significant.

\section{RESULTS}

\section{Liquid extraction yields and qualitative phytochemical screening}

Extraction of $1 \mathrm{~g}$ quantities of dried plant material with various solvents yielded dried plant extracts ranging from $64 \mathrm{mg}$ (ethyl acetate extract) to $276 \mathrm{mg}$ (methanolic extract; Table 1). Methanol and water gave relatively high yields of dried extracted material (276 and $253 \mathrm{mg}$ respectively), whilst ethyl acetate, chloroform and hexane extracted substantially lower masses $(64,165$ and $79 \mathrm{mg}$, respectively). The dried extracts were resuspended in $10 \mathrm{~mL}$ of deionised water (containing 1\% DMSO), resulting in the extract concentrations shown in Table 1. Phytochemical studies showed that methanol and water extracted the widest range and greatest quantity of phytochemicals in this study. Both extracts showed moderate to high levels of phenolic, saponins, triterpenoids, flavonoids and tannins. Similar classes of phytochemicals were detected in the ethyl acetate and chloroform extracts, although at substantially lower levels.

\section{Antibacterial activity}

To examine the growth inhibitory activity of the G. glabra root extracts, a series of disc diffusion assays were conducted on nutrient agar plates inoculated with autoimmune inflammatory disease initiating bacterial strains (P. mirabilis, K. pneumoniae, A. baylyi and P. aeruginosa). P. mirabilis growth was only inhibited by the methanolic and chloroform G. glabra root extracts (Figure 1), with zones of inhibition of $7.2 \pm 0.3$ and 6.3 $\pm 0.2 \mathrm{~mm}$ respectively. These zones of inhibition are indicative of low to moderate growth inhibition. However, this bacterial strain was also somewhat resistant to penicillin and completely resistant to nystatin. Furthermore, previous studies have also highlighted the resistance of this strain against several other conventional antibiotics. ${ }^{4,28,29}$ Therefore, these extracts may still have potential for the prevention and treatment of rheumatoid arthritis in genetically susceptible people. In contrast, ciprofloxacin and chloramphenicol were potent inhibitors of $P$. mirabilis growth. No growth inhibitory activity was observed for the aqueous, ethyl acetate or hexane extracts.

The G. glabra root extracts were substantially more effective inhibitors of K. pneumoniae (the bacterial trigger of ankylosing spondylitis; Figure 2). All extracts inhibited K. pneumoniae growth, although the methanolic and aqueous extracts were particularly good inhibitors, with zones of inhibition of $9.3 \pm 0.6$ and $10.0 \pm 1.0 \mathrm{~mm}$ respectively. This is a noteworthy result as the $K$. pneumoniae strain tested in these studies was completely resistant to penicillin and nystatin and has previously been reported to be resistant to multiple other antibiotics. ${ }^{4,28,29}$ Thus, these extracts have potential for the prevention and treatment of ankylosing spondylitis in genetically susceptible people. This bacterium was also highly susceptible to chloramphenicol and ciprofloxacin, with zones of inhibition between 22 and $25 \mathrm{~mm}$. Substantially lower growth inhibitory activity was noted for the mid to lower polarity ethyl acetate, chloroform and hexane extracts, each with zones of inhibition $<7.5 \mathrm{~mm}$.

The G. glabra root extracts produced mixed results against the bacterial triggers of multiple sclerosis ( $A$. baylyi and P. aeruginosa; Figure 3 ). The methanolic extract was a strong inhibitor of $A$. baylyi growth, producing an inhibition zone of $10 \pm 1.0 \mathrm{~mm}$ (Figure $3 \mathrm{a}$ ). The ethyl acetate extract was also a moderate inhibitor of $A$. baylyi growth $(7.6 \pm 0.3 \mathrm{~mm}$ inhibition zone). Whist the aqueous, chloroform and hexane extracts also inhibited A. baylyi growth, the relatively small zones of inhibition indicated only weak activity. However, it is noteworthy that the A. baylyi strain tested in this study was completely resistant to penicillin and nystatin. Thus, these extracts may still be useful for the prevention and treatment of multiple sclerosis in genetically susceptible people. In contrast, all the G. glabra root extracts were completely devoid of growth inhibitory activity against $P$. aeruginosa (Figure $3 \mathrm{~b}$ ).

\section{Quantification of minimum inhibitory concentration (MIC)}

The minimum inhibitory concentration for each extract was determined by further analysing the extracts that showed antimicrobial activity against susceptible bacterial strains in the disc diffusion assays. The G. glabra root extracts were tested across a range of concentrations against A. baylyi, P. mirabilis, K. pneumoniae and P. aeruginosa in microplate 
Table 1: The mass of dried extracted material, the concentration after resuspension in deionised water (containing $1 \%$ DMSO) and qualitative phytochemical screenings of the G. glabra root extracts.

\begin{tabular}{|c|c|c|c|c|c|c|c|c|c|c|c|c|c|c|c|}
\hline 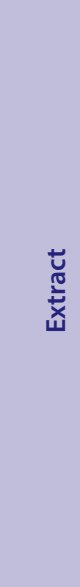 & 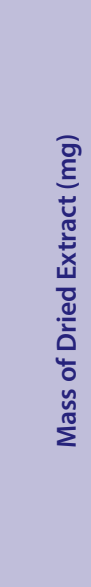 & 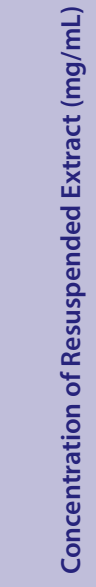 & $\begin{array}{l}\frac{\tilde{U}}{0} \\
\frac{0}{0} \\
\frac{\tilde{\sigma}}{0} \\
\frac{\pi}{0} \\
\stackrel{0}{0}\end{array}$ & 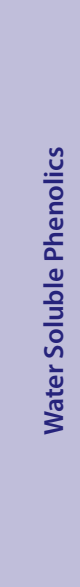 &  & 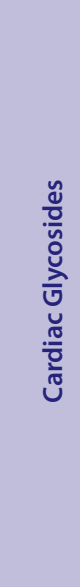 & 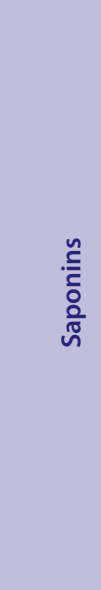 & 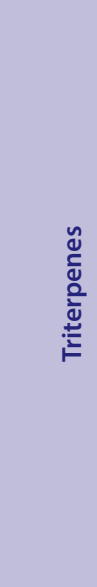 & 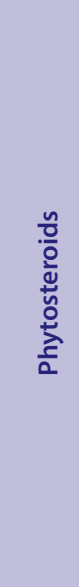 & 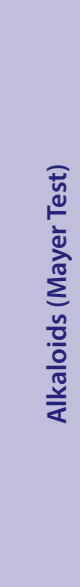 & 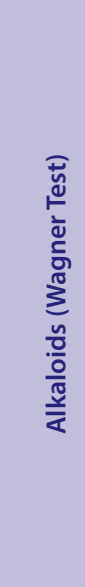 & $\begin{array}{l}\frac{n}{0} \\
\frac{0}{0} \\
\frac{0}{0} \\
\frac{\pi}{4}\end{array}$ & 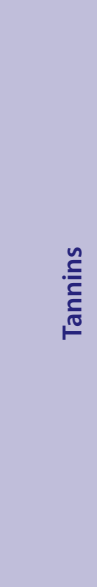 & 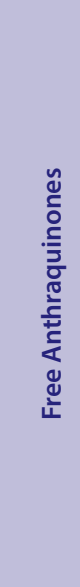 & 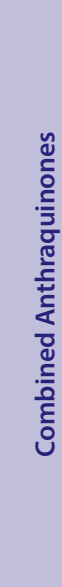 \\
\hline M & 276 & 27.6 & +++ & ++ & +++ & + & +++ & +++ & - & + & + & +++ & +++ & - & - \\
\hline W & 253 & 25.3 & +++ & ++ & +++ & + & +++ & ++ & - & + & + & +++ & +++ & - & - \\
\hline $\mathrm{E}$ & 64 & 6.4 & ++ & + & + & - & ++ & ++ & - & - & - & ++ & + & - & - \\
\hline $\mathrm{C}$ & 165 & 16.5 & + & - & - & - & ++ & ++ & - & - & - & + & + & - & - \\
\hline $\mathrm{H}$ & 79 & 7.9 & + & - & - & - & - & + & - & - & - & - & - & - & - \\
\hline
\end{tabular}

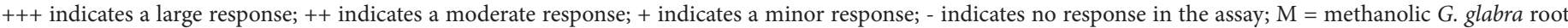
extract; W = aqueous $G$. glabra root extract; E = ethyl acetate G. glabra root extract; C = chloroform G. glabra root extract; H = hexane G. glabra root extract.

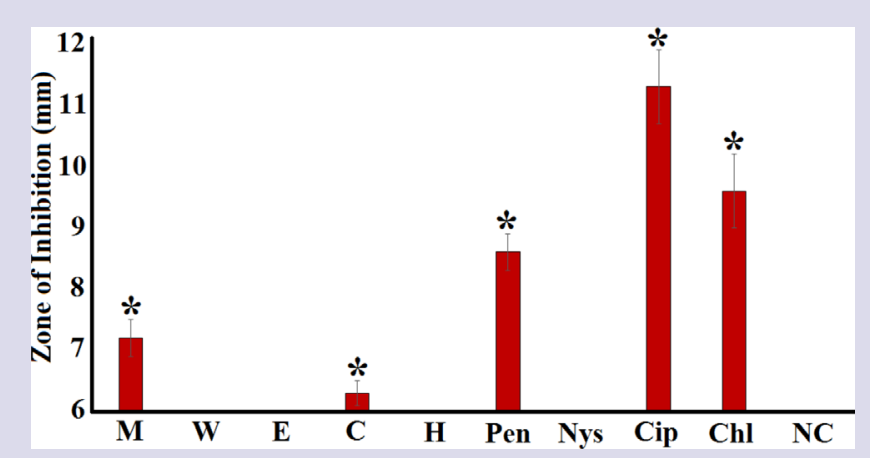

Figure 1: Antibacterial activity of the G. glabra root extracts against P. mirabilis (ATCC: 21721) measured as zones of inhibition ( $\mathrm{mm}$ ). $\mathrm{M}=$ methanolic extract; $\mathrm{W}=$ aqueous extract; $\mathrm{E}=$ ethyl acetate extract; $\mathrm{C}=$ chloroform extract; $\mathrm{H}=$ hexane extract. Positive controls:

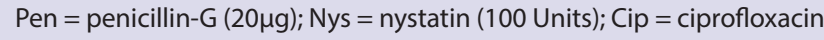
$(2.5 \mu \mathrm{g}) ; \mathrm{Chl}=$ chloramplenicol $(10 \mu \mathrm{g})$. Negative control $(\mathrm{NC})=$ water. Results are expressed as mean zones of inhibition of at least six replicates (two repeats) \pm SEM. * indicates results that are significantly different to the negative control $(\mathrm{P}<0.01)$.

liquid dilution and disc diffusion assays to determine the MIC of each extract (Table 2). MIC values for the antibiotic controls are only provided for the liquid dilution assays as the standard antibiotic control discs used for the disc diffusion assay were only tested at a single dose. The aqueous and ethyl acetate extracts displayed substantially lower MIC values than the other extracts against K. pneumoniae and A. baylyi. Indeed, the ethyl acetate extract had MIC values substantially $<500 \mu \mathrm{g} / \mathrm{mL}$ for both

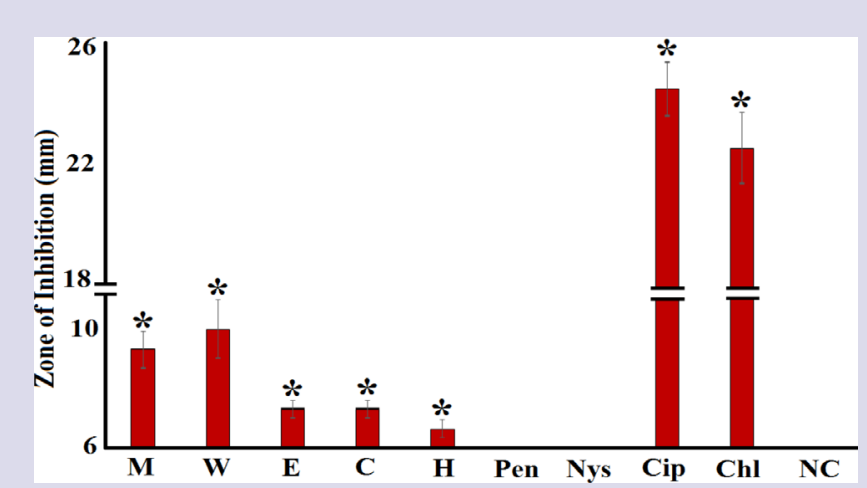

Figure 2: Antibacterial activity of the G. glabra root extracts against K. pneumoniae (ATCC: 39324$)$ measured as zones of inhibition (mm). $\mathrm{M}=$ methanolic extract; $\mathrm{W}=$ aqueous extract; $\mathrm{E}=$ ethyl acetate extract; $\mathrm{C}=$ chloroform extract $\mathrm{H}=$ hexane extract. Positive controls:

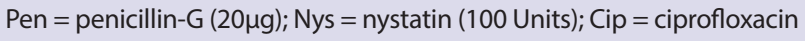
$(2.5 \mu \mathrm{g}) ; \mathrm{Chl}=$ chloramplenicol $(10 \mu \mathrm{g})$. Negative control (NC) = water. Results are expressed as mean zones of inhibition of at least six replicates (two repeats) \pm SEM. ${ }^{*}$ indicates results that are significantly different to the negative control $(P<0.01)$.

bacteria, indicating its potential in the prevention and treatment of ankylosing spondylitis and multiple sclerosis. The aqueous extract was also a strong inhibitor of $K$. pneumoniae growth but had only low activity against $A$. baylyi. Both the aqueous and ethyl acetate extracts were completely devoid of inhibitory activity against $P$. mirabilis and $P$. aeruginosa. The methanolic extract was a moderate inhibitor of all of the bacteria tested except $P$. aeruginosa, whilst the MIC values determined for the 


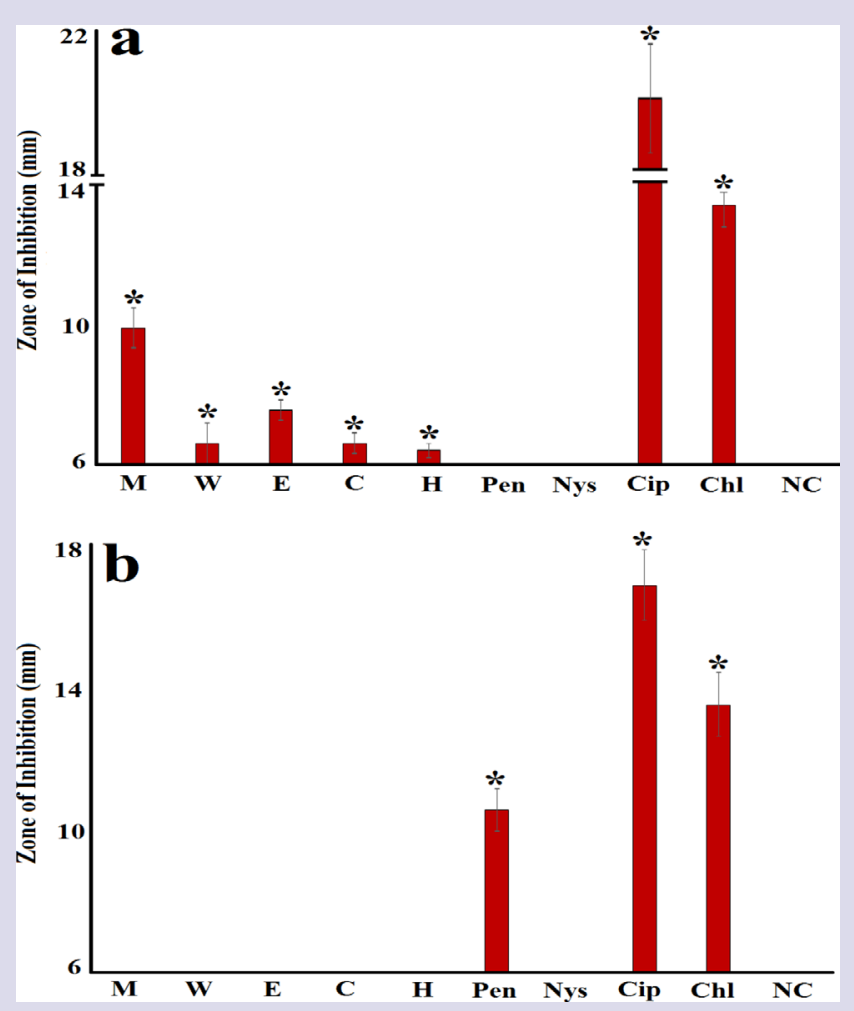

Figure 3: Antibacterial activity of the G. glabra root extracts against (a) A. baylyi (ATCC:21721) and (b) P. aeruginosa (ATCC:31488) measured as zones of inhibition $(\mathrm{mm}) \cdot \mathrm{M}=$ methanolic extract; $\mathrm{W}=$ aqueous extract; $\mathrm{E}=$ ethyl acetate extract; $\mathrm{C}=$ chloroform extract; $\mathrm{H}=$ hexane extract. Positive controls: Pen = penicillin-G $(20 \mu \mathrm{g}) ;$ Nys $=$ nystatin (100 Units); Cip = ciprofloxacin $(2.5 \mu \mathrm{g}) ; \mathrm{Chl}=$ chloramplenicol $(10 \mu \mathrm{g})$. Negative control (NC) = water. Results are expressed as mean zones of inhibition of at least six replicates (two repeats) \pm SEM. * indicates results that are significantly different to the negative control $(P<0.01)$.

chloroform and hexane extracts indicate low growth inhibitory activity against some bacteria, and no activity against others.

\section{Determination of combinational effects: Fractional Inhibitory Concentration (FIC) assessment}

Fractional inhibitory concentration (FIC) determination was performed using a 1:1 ratio of each $G$. glabra root extract to conventional antibiotic and sums of FIC ( $\Sigma$ FIC) were calculated for any combinations containing extracts that inhibited bacterial growth on their own (Table 3). The combination containing the aqueous G. glabra root extract and chloramphenicol tested against $K$. pneumoniae was the only synergistic interaction detected $(\Sigma F I C=0.38)$. This is an interesting result as this bacterium was resistant against chloramphenicol alone (MIC $2.5 \mu \mathrm{g} / \mathrm{mL}$ ). Further studies are warranted to examine the synergistic mechanism and to identify the synergising component(s) in the extracts. In addition, 13 additive interactions were detected. Whilst not as promising as the synergistic combination, these combinations do have substantially enhanced activity compared to the individual components when tested alone and thus warrant further investigation. Seven non-interactive combinations were also detected. Whilst these combinations have no extra beneficial effects against these bacteria, the extracts do not counteract the actions of the conventional antibiotics and so they are safe to use in combination. Although $\Sigma$ FIC values could not be determined for the remaining combinations
(Table 3), it is noteworthy that no antagonistic interactions were detected, indicating that all of the G. glabra root extracts can be used in combination with the conventional antibiotics tested in this study without reducing the effects of the antibiotic component of the combinations.

\section{Varied ratio combination studies (isobolograms)}

As a synergistic interaction was detected for the aqueous G. glabra root extract: chloramphenicol combination against $K$. pneumoniae, ratios of this combination were tested in order to identify the optimal ratios at which synergy occurs (Figure 4). Of the nine ratios of the aqueous G. glabra root extract in combination with chloramphenicol, three were synergistic (40-60\% extract). Therefore, all of these ratios would be effective for inhibiting the growth of $K$. pneumoniae. The other six ratios yielded additive interactions. The ideal synergistic ratio for the treatment and prevention of ankylosing spondylitis was therefore identified to be $60 \%$ G. glabra aqueous root extract and $40 \%$ chloramphenicol, as this ratio would minimise the amount of chloramphenicol in the combination and thus reduce the chances of developing further resistance with long term prophylactic usage. In contrast, the preferred ratio for acute infections (the highest antibiotic \% to maximise the efficacy of the treatment) is the $40 \%$ G. glabra aqueous root extract and $60 \%$ chloramphenicol ratio.

\section{Quantification of toxicity}

To evaluate and quantify the effect of the extracts on the induction of mortality, each was diluted in artificial seawater to test across a range of concentrations in the Artemia nauplii bioassay. Table 4 shows the $\mathrm{LC}_{50}$ values of the extracts towards $A$. franciscana. No $\mathrm{LC}_{50}$ values are reported for the chloroform, hexane and ethyl acetate extracts as $<50 \%$ mortality was seen for all concentrations tested. The methanolic and aqueous extracts were also determined to be nontoxic, with $\mathrm{LC}_{50}$ values substantially greater than $1000 \mu \mathrm{g} / \mathrm{mL}$ following $24 \mathrm{~h}$ exposure. Extracts with an $\mathrm{LC}_{50}$ of greater than $1000 \mu \mathrm{g} / \mathrm{mL}$ towards Artemia nauplii have been defined as being nontoxic. ${ }^{25}$ Similarly, the HDF cell viability was $>50 \%$ for all extract treatments, confirming that all extracts were nontoxic.

\section{DISCUSSION}

This study examined the growth inhibitory activity of G. glabra root extracts against selected bacterial triggers of autoimmune inflammatory diseases, both alone and in combination with conventional antibiotics. The high to mid polarity methanol, water and ethyl acetate G. glabra extracts were good bacterial growth inhibitors, whereas the less polar chloroform and hexane extracts had substantially lower growth inhibitory activity. The ethyl acetate extract was a particularly potent inhibitor of K. pneumoniae and A. baylyi growth, with MIC values of approximately $450 \mu \mathrm{g} / \mathrm{mL}$ against both species. The aqueous extract was similarly potent against $K$. pneumoniae but was only a low to moderate inhibitor of A. baylyi. As K. pneumoniae is a bacterial trigger of ankylosing spondylitis in genetically susceptible people, ${ }^{15}$ these extracts have potential for preventing and treating that disease. Furthermore, the ethyl acetate extract has therapeutic potential for the treatment and prevention of multiple sclerosis as A. baylyi can trigger that disease in genetically individuals. ${ }^{15}$ However, as $P$ aeruginosa is a further trigger of multiple sclerosis and the G. glabra extracts were completely devoid of inhibitory activity against that bacteria, it is likely that the G. glabra ethyl acetate extract will have limited efficacy as a preventative therapy for multiple sclerosis in vivo and further studies are required to examine this.

Whilst an examination of the phytochemistry of the G. glabra root extracts was beyond the scope of our study, high relative abundances of polyphenolics, saponins, triterpenoids, flavonoids and tannins were detected in the qualitative phytochemical screening studies. This is consistent with previous studies which have reported that G. glabra extracts 
Table 2: Disc diffusion and liquid dilution MIC values $(\mu \mathrm{g} / \mathrm{mL})$ against the bacterial triggers of some selected autoimmune inflammatory diseases.

\begin{tabular}{|c|c|c|c|c|c|c|c|c|}
\hline \multirow{3}{*}{ Extract } & \multicolumn{8}{|c|}{ Minimum Inhibitory Concentration ( $\mu \mathrm{g} / \mathrm{mL}$ ) } \\
\hline & \multicolumn{2}{|c|}{ P. mirabilis } & \multicolumn{2}{|c|}{ K. pneumoniae } & \multicolumn{2}{|c|}{ A. baylyi } & \multicolumn{2}{|c|}{ P. aeruginosa } \\
\hline & DD & LD & DD & LD & DD & LD & $\mathrm{DD}$ & LD \\
\hline Methanol & 1950 & 1521 & 1478 & 1219 & 1204 & 760 & - & - \\
\hline Water & - & - & 725 & 981 & 3506 & 4910 & - & - \\
\hline Ethyl acetate & - & - & 453 & 925 & 425 & 463 & - & - \\
\hline Chloroform & 6750 & 3245 & 6387 & 3245 & $>10,000$ & 4625 & - & - \\
\hline Hexane & - & - & 5322 & 2716 & 7805 & 5308 & - & - \\
\hline \multicolumn{9}{|l|}{ Positive control } \\
\hline Penicillin & ND & 2.5 & ND & 3.3 & ND & 3.3 & ND & 3.3 \\
\hline Erythromycin & ND & 3.3 & ND & 1.9 & ND & 2.5 & ND & 3.3 \\
\hline Chloramphenicol & ND & 2.5 & ND & 2.5 & ND & 1.63 & ND & 2.5 \\
\hline Tetracycline & ND & 1.25 & ND & 1.9 & ND & 1.25 & ND & 2.5 \\
\hline
\end{tabular}

The values represent the MIC value in $\mu \mathrm{g} / \mathrm{mL}$. $\mathrm{DD}=$ disc diffusion; $\mathrm{LD}=$ liquid dilution; $\mathrm{ND}=$ not determined; - = no inhibition was observed.

\begin{tabular}{|c|c|c|c|c|c|}
\hline \multirow{2}{*}{ Bacteria } & \multirow{2}{*}{ Extract } & \multicolumn{4}{|c|}{ 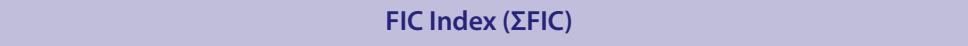 } \\
\hline & & Penicillin & Erythromycin & Chloramphenicol & Tetracycline \\
\hline \multirow{5}{*}{ 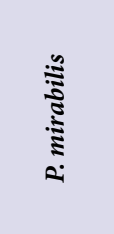 } & M & $\mathrm{CND}$ & CND & CND & CND \\
\hline & $\mathrm{W}$ & $\mathrm{CND}$ & CND & 0.556 & $\mathrm{CND}$ \\
\hline & $\mathbf{E}$ & CND & CND & CND & CND \\
\hline & $\mathrm{C}$ & CND & CND & CND & CND \\
\hline & $\mathbf{H}$ & $\mathrm{CND}$ & CND & CND & CND \\
\hline \multirow{5}{*}{ 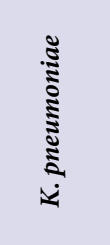 } & $\mathbf{M}$ & CND & CND & 1.01 & CND \\
\hline & W & CND & CND & 0.49 & $\mathrm{CND}$ \\
\hline & $\mathbf{E}$ & CND & CND & 1.01 & CND \\
\hline & C & CND & CND & CND & CND \\
\hline & $\mathbf{H}$ & CND & CND & CND & CND \\
\hline \multirow{5}{*}{$\begin{array}{l}\tilde{7} \\
\frac{\pi}{5} \\
\dot{\nabla}\end{array}$} & $\mathbf{M}$ & 0.505 & CND & CND & 0.505 \\
\hline & $\mathrm{W}$ & 1.01 & CND & CND & 0.603 \\
\hline & $\mathbf{E}$ & 0.505 & CND & CND & $\mathrm{CND}$ \\
\hline & C & 0.603 & CND & CND & 0.596 \\
\hline & $\mathbf{H}$ & CND & CND & CND & CND \\
\hline \multirow{5}{*}{ 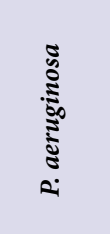 } & $\mathbf{M}$ & CND & 1.01 & CND & 1.01 \\
\hline & W & CND & 0.508 & CND & 0.505 \\
\hline & $\mathbf{E}$ & CND & 0.505 & CND & 1.01 \\
\hline & C & $\mathrm{CND}$ & 0.96 & CND & 1.01 \\
\hline & $\mathbf{H}$ & CND & 0.508 & CND & 0.505 \\
\hline
\end{tabular}

Numbers indicate the mean $\Sigma$ FIC values of 6 determinations; $C N D=$ could not be determined as one or both components of the combination were inactive; Synergy (blue highlighting) $=\Sigma$ FIC $\leq 0.5$; Additive $=>0.5-\leq 1.0$; Indifferent effects $=>1.0-\leq 4.0$.

contain high levels of triterpenoid saponins including glycyrrhizic acid (Figure 5a) and glycyrrhetinic acid (Figure 5b). Indeed, triterpenoid saponins account for up to $20 \%$ of dried root mass. ${ }^{8}$ The root was also rich in flavonoids. This is also consistent with previous studies which have reported the presence of high levels of flavonoids and isoflavones, including glabridin (Figure 5c), glabrone (Figure 5d), O-methyl-glabridin
(Figure 5e), 3'-hydroxy-4'-methylglabridin (Figure 5f), hispalabridin A (Figure $5 \mathrm{~g}$ ), and hispalabridin B (Figure $5 \mathrm{~h}$ ) in G. glabra extracts. ${ }^{8}$ Interestingly, several of these G. glabra flavonoids have been reported to inhibit the growth of multiple bacterial pathogens. ${ }^{10-14}$ Therefore, it is likely that the G. glabra flavonoids contribute to the inhibitory activity recorded in our study. 




Figure 4: Isobologram for combinations of chloramphenicol and aqueous G. glabra root extracts tested at various ratios against $K$. pneumoniae (ATCC: 39324). Results represent mean MIC values of four replicates. Ratio $=\%$ extract: \% antibiotic. Ratios lying on or underneath the $0.5: 0.5$ (green) line are synergistic ( $\Sigma \mathrm{FIC} \leq 0.5)$. Any points between the $0.5: 0.5$ (green) and 1.0:1.0 (blue) line or on the 1.0:1.0 (orange) line are deemed additive $(\Sigma \mathrm{FIC}>0.5-1.0)$.

Table 4: Toxicity determination of the G. glabra root extracts in the Artemia nauplii (ALA) and human dermal fibroblast (HDR) assay following $24 \mathrm{~h}$ exposure.

\begin{tabular}{ccc}
\hline \multirow{2}{*}{ Extract } & \multicolumn{2}{c}{ Toxicity } \\
\cline { 2 - 3 } & ALA $(\mu \mathrm{g} / \mathrm{mL})$ & HDF assay \\
\hline Methanol & 1650 & NT \\
Water & 1983 & NT \\
Ethyl acetate & - & NT \\
Chloroform & - & NT \\
Hexane & - & NT
\end{tabular}

- indicates that $<50 \%$ mortality was induced at all concentrations tested. NT $=$ not toxic ( $>50 \%$ cell viability) in the HDR assay.

Other phytochemicals may also contribute to the antibacterial activity of the G. glabra extracts. G. glabra is reportedly rich in chalconoids including licochalcone A (Figure 5i), licochalcone B (Figure 5j), licochalcone D (Figure 5k), licochalcone C (Figure 5l), isoliquiritigen (Figure $5 \mathrm{~m}$ ) and echinatin (Figure 5n), ${ }^{8}$ and these compounds may contribute to the antibacterial activity. Furthermore, the qualitative phytochemical screens in our study detected high levels of tannins in the mid to high polarity G. glabra extracts. This is interesting as tannins are known to be potent antibacterial agents. Gallotannins have been reported to inhibit the growth of a broad spectrum of bacterial species ${ }^{30}$ through a variety of mechanisms including binding lipotoichoic acid and proline-rich cell surface proteins, ${ }^{31,32}$ and by inhibiting glucosyltransferase enzymes. ${ }^{33}$ Elligitannins are also highly potent inhibitors of bacterial growth, with MIC values as low as $62.5 \mu \mathrm{g} / \mathrm{ml} .{ }^{30}$ Ellagitannins have also been reported to function via several antibiotic mechanisms including interaction with cytoplasmic oxidoreductases and by disrupting bacterial cell walls..$^{30,32}$

Although inhibition of the growth of some bacterial triggers of autoimmune inflammatory diseases was noteworthy, the results of the combinational

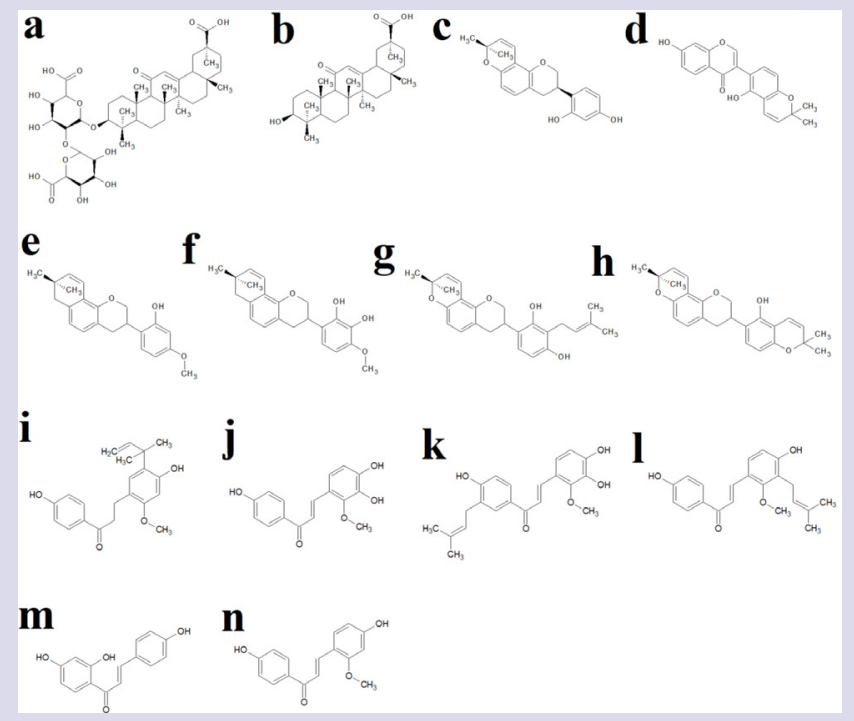

Figure 5: Chemical strudctures of (a) glycyrrhizic acid, (b) glycyrrhetinic acid, (c) glabridin, (d) glabrone, (e) O-methyl-glabridin, (f) 3'-hydroxy-4'methylglabridin, (g) hispalabridin A, (h) hispalabridin B, (i) licochalcone $A$, (j) licochalcone $B$, (k) licochalcone $D$, (I) licochalcone $C,(m)$ isoliquiritigen, $(n)$ echinatin.

studies were perhaps of greater significance. The combination of the ethyl acetate G. glabra extract and chloramphenicol displayed substantially greater growth inhibitory activity against K. pneumoniae than either the extracts or conventional antibiotics alone, highlighting possible future therapies for ankylosing spondylitis. Indeed, when used in combination with chloramphenicol, the ethyl acetate extract induced synergistic interactions against $K$. pneumoniae. Synergistic interactions allow for greater efficacy in drug administration by increasing a drug's effectiveness or reduce the potential for the development of further microbial resistance by allowing lower doses of the conventional antibiotic to be prescribed. ${ }^{23}$ Furthermore, synergistic combinations of the G. glabra extracts and conventional antibiotics could potentially repurpose antibiotics which would otherwise be ineffective against resistant bacterial strains. Examples of combinational medicines repurposing conventional antibiotics already exist. The drug Augmentin takes advantage of the synergising activity of clavulanic acid in combination with $\beta$-lactam antibiotics. ${ }^{1}$ Clavulanic acid alone has negligible inherent antibacterial activity. However, it binds irreversibly to bacterial $\beta$-lactamase enzymes, thereby inactivating them and overcoming bacterial resistance to $\beta$-lactams. ${ }^{1}$ Research has identified several strains of $K$. pneumoniae that possess resistance to a wide array of antibiotics of several classes. ${ }^{34}$ Our data from the antimicrobial screening was consistent with these studies as penicillin-G, erythromycin, chloramphenicol and tetracycline were all relatively ineffective growth inhibitors of K. pneumoniae. Future studies focusing on the mechanism of synergy are required.

Whilst only one synergistic interaction was detected, a further thirteen additive combinations were noted, with approximately 50\% of those against $P$. aeruginosa. These combinations would also be beneficial in the prevention and treatment of the autoimmune inflammatory diseases. Seven combinations provided indifferent interactions. Whilst administration of these combinations would not provide any benefit above that of the antibiotic alone, they also would not lessen the activity of the conventional antibiotics. Interestingly, no antagonistic combinations were detected, further indicating that the extract-conventional antibiotic 
combinations can be safely used without lessening the antibiotics effects. This is an important finding as many people self-medicate with herbal treatments concurrently with allopathic medicines.

\section{CONCLUSION}

Mid to high polarity G. glabra root extracts inhibited the growth of K. pneumoniae and A. baylyi when used alone and therefore have potential in the prevention and treatment of ankylosing spondylitis and multiple sclerosis. The G. glabra root ethyl acetate extract also potentiated the activity of chloramphenicol synergistically and thus may provide the framework for future combinational treatments. Chloramphenicol could potentially be repurposed to allow it to inhibit bacteria which would otherwise be unaffected by its actions.

\section{ACKNOWLEDGEMENT}

Financial support for this work was provided by the Environmental Futures Research Institute and the School of Natural Sciences, Griffith University, Australia.

\section{REFERENCES}

1. Cheesman MJ, Ilanko A, Blonk B, Cock IE. Developing new antimicrobial therapies: Are synergistic combinations of plant extracts/compounds with conventional antibiotics the solution? Pharmacognosy Reviews. 2017;11(22):57-72. DOI: 10.4103/phrev.phrev_21_17.

2. WHO. Antimicrobial Resistance. World Health Organization. 2016. Available from: http://www.who.int/mediacentre/factsheets/fs194/en/. [Last cited on 2017 May 10]

3. Sirdaarta J, Matthews B, Cock IE. Kakadu plum fruit extracts inhibit the growth of the bacterial triggers of rheumatoid arthritis: Identification of stilbene and tannin components. Journal of Functional Food. 2015;17:610-20. DOI: 10.1016/j. jff.2015.06.019.

4. Ilanko A, Cock IE. The interactive antimicrobial activity of contentional antibiotics and Petalostigma spp. Extracts against bacterial triggers of some autoimmune inflammatory diseases. BMC Complementary and Alternative Medicine. 2018; in press.

5. Chowdhury AN, Ashrafuzzaman M, Ali H, Liza LN, Zinnah KMA. Antimicrobial activity of some medicinal plants against multi drug resistant human pathogens. Advances in Bioscience and Bioengineering. 2013;1(1):1-24.

6. Zargaran A, Zarshenas MM, Mehdizadeh A, Mohagheghzadeh A. Management of tremor in medieval Persia. Journal of the History of Neurosciences. 2013 22(1):53-61.

7. Esmaeili S, Naghibi F, Mosaddegh M, Sahranavard S, Ghafari S, Abdullah NR. Screening of antiplasmodial properties among some traditionally used Iranian plants. Journal of Ethnopharmacology. 2009;121(3):400-4.

8. Asl MN, Hosseinzadeh $\mathrm{H}$. Review of pharmacological effects of Glycyrrhiza sp. and its bioactive compounds. Phytotherapy Research. 2008;22(6):709-24.

9. Sabahi M, Mansouri SH, Ramezanian M, Gholam-Hoseinian A. Screening of plants from the southeast of Iran for antimicrobial. International Journal of Crude Drug Research. 1987;25(2):72-6.

10. Hatano T, Shintani Y, Aga Y, Shiota S, Tsuchiya T, Yoshida T. Phenolic constituents of licorice. VIII. Structures of glicophenone and glicoisoflavanone, and effects of licorice phenolics on methicillin-resistant Staphylococcus aureus. Chemical and Pharmaceutical Bulletin. 2000;48(9):1286-92.

11. Hatano T, Kusuda M, Inada K, Ogawa TO, Shiota S, Tsuchiya T, et al. Effects of tannins and related polyphenols on methicillin-resistant Staphylococcus aureus. Phytochemistry. 2005;66(17):2047-55.

12. Fukai T, Marumo A, Kaitou K, Kanda T, Terada S, Nomura T. Anti-Helicobacter pylori flavonoids from licorice extract. Life Sciences. 2002;71(12):1449-63.

13. Fukai T, Marumo A, Kaitou K, Kanda T, Terada S, Nomura T. Antimicrobial activity of licorice flavonoids against methicillin-resistant Staphylococcus aureus. Fitoterapia. 2002;73(6):536-9.
14. Onkarappa R, Shobha KS, Chaya K. Efficacy of four medicinally important plant extracts (crude) against pathogenic bacteria. Asian Journal of Microbiology, Biotechnology and Environmental Sciences. 2005;7(2):281-4.

15. Courtney R, Sirdaarta J, Matthews B, Cock IE. Tannin components and inhibitory activity of Kakadu plum leaf extracts against microbial triggers of autoimmune inflammatory diseases. Pharmacognosy Journal. 2015;7(1):18-31. DOI: 10.5530/pj.2015.7.2

16. Cock IE, Cheesman M. The potential of plants of the genus Syzygium (Myrtaceae) for the prevention and treatment of arthritic and autoimmune diseases. In Bioactive Foods as Dietary Interventions for Arthritis, Osteoarthritis, and Related Autoimmune Siseases. $2^{\text {nd }}$ edition. 2018. Edited by Preedy VR, Watson RR; Elsevier, Netherlands.

17. Boyer $\mathrm{H}$, Cock IE. Evaluation of the potential of Macademia integriflora extracts as antibacterial food agents. Pharmacognosy Communications. 2013;3(3):53-62. DOI: $10.5530 / p c .2013 .3 .10$

18. Vesoul J, Cock IE. The potential of Bunya nut as an antibacterial food agent Pharmacognosy Communications. 2012;2(1):72-9. DOI: 10.5530/pc.2012.1.13

19. Winnett $\mathrm{V}$, Boyer H, Sirdaarta J, Cock IE. The potential of Tasmannia lanceolata as a natural preservative and medicinal agent: Antimicrobial activity and toxicity. Pharmacognosy Communications. 2014;4(1):42-52. DOI: 10.5530/pc.2014.1.7

20. Cock IE, Van Vuuren SF. Anti-Proteus activity of some South African medicinal plants: Their potential for the treatment and prevention of rheumatoid arthritis. Inflammopharmacology. 2014;22(1):23-36. DOI 10.1007/s10787-013-0179-3.

21. Cock IE. Antimicrobial activity of Acacia aulacocarpa and Acacia complanta methanolic extracts. Pharmacognosy Communications. 2012;2(1):66-71. DOI: 10.5530/pc.2012.1.12

22. Eloff JN. A Sensitive and quick microplate method to determine the minimal inhibitory concentration of plant extracts for bacteria. Planta Medica. 1998; 64(8):711-3.

23. Hubsch Z, Van Zyl RL, Cock IE, Van Vuuren SF. Interactive antimicrobial and toxicity profiles of conventional antimicrobials with Southern African medicinal plants. South African Journal of Botany. 2014;93:185-97. DOI: 10.1016/j. sajb.2014.04.005.

24. Arkhipov A, Sirdaarta J, Rayan P, McDonnell PA, Cock IE. An examination of the antibacterial, antifungal, anti-Giardial and anticancer properties of Kigelia africana fruit extracts. Pharmacognosy Communications. 2014;4(3):62-76. DOI: 10.5530/pc.2014.3.7.

25. Ruebhart DR, Wickramasinghe W, Cock IE. Protective efficacy of the antioxidants vitamin $\mathrm{E}$ and Trolox ${ }^{\mathrm{TM}}$ against Microcystis aeruginosa, microcystin-LR and menadione toxicity in Artemia franciscana nauplii. Journal of Toxicology and Environmental Health Part A. 2009;72(24):1567-75.

26. Vesoul J, Cock IE. An examination of the medicinal potential of Pittosporum phylloraeoides: Toxicity, antibacterial and antifungal activities. Pharmacognosy Communications. 2011;1(2):8-17. DOI: 10.5530/pc.2011.2.3

27. Shalom J, Cock IE. Terminalia ferdinandiana Exell. fruit and leaf extracts inhibit proliferation and induce apoptosis in selected human cancer cell lines. Nutritrion and Cancer. 2018. DOI: 10.1080/01635581.2018.1460680.

28. Mandeville A, Cock IE. Terminalia chebula Retz. Fruit extracts inhibit some bacterial triggers of autoimmune inflammatory diseases and potentiate the activity of tetracycline. Pharmacognosy Journal. 2018. in press.

29. Hutchings A, Cock IE. The interactive antimicrobial activity of Embelica officinalis Gaertn. Fruit extracts and conventional antibiotics against some bacterial triggers of autoimmune inflammatory diseases. Pharmacognosy Journal. 2018. in press.

30. Buzzini P, Arapitsas P, Goretti M, Branda E, Turchetti B, Pinelli P, et al. Antimicrobial activity of hydrolysable tannins. Mini-Reviews in Medicinal Chemistry. 2008;8(12):1179-87.

31. Wolinsky LE, Sote EO. Isolation of natural plaque-inhibiting substances from 'Nigerian chewing sticks'. Caries Research. 1984;18(3):216-25.

32. Hogg SD, Embery G. Blood-group-reactive glycoprotein from human saliva interacts with lipoteichoic acid on the surface of Streptococcus sanguis cells. Archives in Oral Biology. 1982;27(3):261-8.

33. Wu-Yuan CD, Chen CY, Wu RT. Gallotannins inhibit growth, water-soluble glucan synthesis, and aggregation of Streptococci mutans. Journal of Dental Research. 1988;67(1):51-5.

34. Parveen RM, Khan MA, Menezes GA, Harish BN, Parija SC, Hays JP. Extendedspectrum $\beta$-lactamase producing Klebsiella pneumoniae from blood cultures in Puducherry, India. The Indian Journal of Medical Research. 2011;134(3):392-5. 
PICTORIAL ABSTRACT

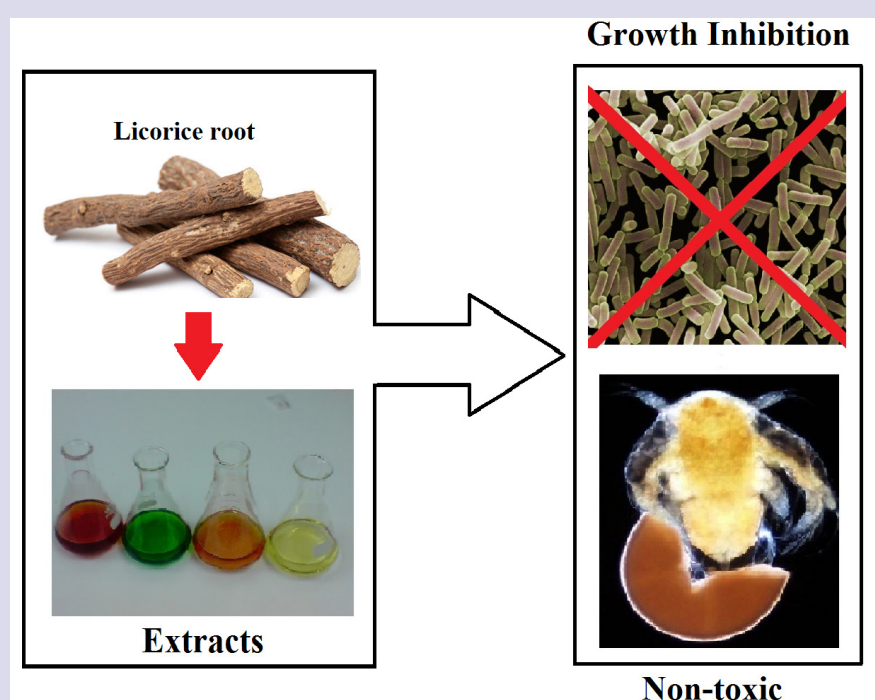

\section{HIGHLIGHTS OF PAPER}

- Methanolic, aqueous and ethyl acetate G. glabra root extracts inhibited the growth of some bacterial triggers of autoimmune diseases

- The ethyl acetate extract was a particularly potent inhibitor of $K$. pneumonia and A. baylyi growth (MICs $<500 \mu \mathrm{g} / \mathrm{mL}$ ).

- The aqueous extract was also a moderate inhibitor of $A$. baylyi growth.

- The aqueous extract also synergised the activity of chloramphenicol against $K$.

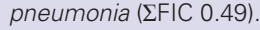

- All G. glabra root extracts were nontoxic in the Artemia and HDF assays.

\section{ABOUT AUTHORS}

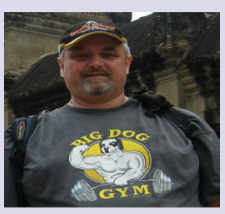

Dr lan Cock leads a research team in the Environmental Futures Research Institute and the School of Natural Sciences at Griffith University, Australia. His research involves bioactivity and phytochemical studies into a variety of plant species of both Australian and international origin, including Aloe vera, South Asian and South American tropical fruits, as well as Australia plants including Scaevola spinescens, Pittosporum phylliraeoides, Terminalia ferdinandiana (Kakadu plum), Australian Acacias, Syzygiums, Petalostigmas and Xanthorrhoea johnsonii (grass trees). This range of projects has resulted in nearly 200 publications in a variety of peer reviewed journals. 